\section{G295(P) FACILITATORS AND BARRIERS OF CONTINUOUS POSITIVE AIRWAY PRESSURE USE IN NEWBORN CARE IN KENYA (PRELIMINARY RESULTS)}

${ }^{1} \mathrm{HM}$ Nabwera, ${ }^{1} \mathrm{~F}$ Dickinson, ${ }^{1} \mathrm{~A}$ Manu, ${ }^{1} \mathrm{P}$ Godia, ${ }^{1,2} \mathrm{MK}$ Sammy, ${ }^{1} \mathrm{BC}$ Naimoi, ${ }^{1} \mathrm{~N}$ van den Broek, ${ }^{1} \mathrm{M}$ Mathai. ${ }^{1}$ Centre for Maternal and Newborn Health, Liverpool School of Tropical Medicine, Liverpool, UK; ${ }^{2}$ Paediatrics, Gertrude's Children's Hospital, Nairobi, Kenya

\subsection{6/archdischild-2018-rcpch.287}

Aim To describe the operational aspects of continuous positive airway pressure (CPAP) use in newborn care in Kenya.

Methods An ongoing nationwide survey of all health facilities in Kenya that use CPAP in newborn care. The mixed method approach used includes a standard questionnaire to describe CPAP use; key informant interviews and focus group discussions with the health care providers, to explore facilitators and barriers to CPAP use in newborn care. Descriptive statistics are used to analyse the quantitative data. A thematic framework is used to analyse the qualitative data.

Results Twenty-two health facilities in Kenya provide CPAP in newborn care, but only 18 (82\%) have agreed to participate in the survey. To date the survey has been conducted in 9 $(50 \%)$ of these facilities, all of which are public county referral facilities that have been using CPAP in for $\leq 2$ years. All of them use commercial bubble CPAP acquired predominantly through donor funding and have $\leq 3$ machines per newborn care unit. Only $7 / 9(78 \%)$ of the newborn care units had a doctor or nurse who had received training on the use of CPAP, and this was often on the job clinical training. The main indications for initiating CPAP were respiratory distress and suspected pneumonia. The main barriers to CPAP use in newborn care were inadequate training of health care providers on the use of CPAP, health care provider strikes and staff shortages, and inadequate support with management of equipment when donor support ended. The main facilitators were good leadership both at the unit and facility level that supported the sustainability of CPAP use in newborn care and peer support from carers whose newborns had survived following CPAP use.

Conclusion The scale-up of CPAP use in newborn care in Kenya is well accepted by health care providers, but there are significant challenges with the sustainability of this intervention. Going forward, implementation strategies need to ensure that all staff working in newborn care units are adequately trained to safely administer CPAP, and that the health facilities are empowered to maintain the CPAP machines or have ready access to this support.

\section{G296(P) IMPLEMENTATION OF A NEONATAL MINIMAL DATASET IN A LOW INCOME COUNTRY}

${ }^{1} \mathrm{C}$ Battersby, ${ }^{1} \mathrm{~V}$ Loidl, ${ }^{1} \mathrm{R}$ Lloyd-Jones, ${ }^{1} \mathrm{P}$ Toussaint, ${ }^{1} \mathrm{C}$ Hill, ${ }^{2} \mathrm{~V}$ Vasu. ${ }^{1}$ Neonatal Unit, Hopital de la Convention Baptist d'Haiti, Hope Health Action Charity, NGO, Haiti; ${ }^{2}$ Neonatal Unit, William Harvey Hospital, Ashford, UK

\subsection{6/archdischild-2018-rcpch.288}

Aim To embed a neonatal minimal dataset into routine neonatal care in a low income country (LIC) to inform and promote quality improvement (QI).

Method In May 2016, we implemented the collection of a subset of data items held on the National Neonatal Research Database (UK) into a NGO funded neonatal unit in Haiti. Data were recorded at admission, discharge, and daily using standardised forms. Data were analysed for a six month period (1 st May to 31 st October 2016) to determine data completeness and provide feedback to staff for QI.

Results Data completeness was high for gestational age (GA) (98\%), mode of delivery (100\%), origin of admission (100\%), outcome at discharge (94\%) but lower for feeding at discharge (61\%), temperature within an hour of admission (75\%) and cause of death (73\%). 301 babies were admitted in the six month period $(72 \%$ term $\geq 37$ weeks; $11 \% 34$ to $<37$ weeks; $12 \% 30$ to $<34$ weeks; $3 \% 28$ to $<30$ weeks). 58\% were born by vaginal delivery and $41 \%$ caesarean section $(24 \%$ emergency, $17 \%$ elective). $73 \%$ were inborn, $23 \%$ from the community and 3\% from another hospital. 58\% were hypothermic (temp $<36.5^{\circ} \mathrm{C}$ ) and $5 \%$ had a temperature of $<34^{\circ} \mathrm{C}$. Overall mortality rate was $9 \%$; by GA 28 to $<30$ weeks (22\%); 30 to $<34$ weeks (31\%); 34 to $<37$ weeks (9\%); $\geq 37$ weeks (4\%). Among babies born 30 to $<34 \mathrm{w}$, the commonest cause of death was gastrointestinal haemorrhage. Among term babies, the most common cause of death was unknown or hypoxia. 34 babies received CPAP for a median 4 days (interquartile range 2-5). 79\% received some breast milk during admission and $47 \%$ were exclusively breast fed at discharge.

Conclusion Our preliminary data have informed QI programmes aimed to reduce hypothermia on admission and mortality rates. We have shown that although it is feasible to collect a minimal dataset in a LIC setting, completeness of data can be a challenge, and we recommend regular feedback to neonatal staff to reinforce the importance of complete and accurate data to drive improvements in clinical care and outcomes.

\section{G297(P) GUTS, GERMS AND IRON: A SYSTEMATIC REVIEW OF THE EFFECT OF IRON SUPPLEMENTATION AND FORTIFICATION ON DIARRHOEA IN CHILDREN AGED 4 TO 59 MONTHS}

${ }^{1}$ A Ghanchi, ${ }^{2}$ P James. 'GKT School of Medical Education, King's College London, London, UK; ${ }^{2}$ Nutrition Group, London School of Hygiene and Tropical Medicine, London, UK

\subsection{6/archdischild-2018-rcpch.289}

Aims The dramatic results of recent trials have highlighted the possible adverse relationship of iron supplementation on infectious disease mortality. A large body of in-vitro evidence has also shown the reliance of multiple gut-pathogens on high physiological iron concentrations. The administration of oral iron during public health campaigns may stimulate pathogen proliferation at the expense of the host, thus increasing the the risk of diarrhoea. The relationship between iron administration and diarrhoea needs to be established, revisited and contextualised within the context of recent developments in the understanding of both iron regulation and the iron-gut axis.

Methods Randomised control trials of iron supplementation or fortification which reported diarrhoeal outcomes in children aged 4 to 59 months were identified from a systematic search of five databases: Medline, EMBASE, Global Health, Web of Science and Cochrane Central Register of Controlled Trials (CENTRAL). A narrative synthesis of included studies was conducted with study quality assessed using the Cochrane Risk of Bias Tool. The protocol for this review was registered in the International Prospective Register for Systematic Reviews (PROSPERO): CRD42017067297. 
Results 19 studies were extracted, 37\% of which showed an increased incidence of diarrhoea within sub-groups, specifically in infants who were iron replete at baseline. 2 studies reported bloody diarrhoea. No clear relationship between type of intervention or volume of iron administered was observed.

Conclusion This review supports the current World Health Organisation (WHO) guidelines, which recommend the use of iron interventions only in areas with a high prevalence of anaemia. However, the potential unnecessary morbidity burden which may be induced by indiscriminate blanket population interventions must be reconsidered. Using the iron regulating hormone hepcidin, this review proposes a novel mechanism by which iron replete individuals may be at highest risk of diarrhoea as well as future methods of mitigating such adverse outcomes.

\section{G298(P) ZIKA VIRUS ASSOCIATED MENINGOENCEPHALITIS IN CHILDREN: ARE WE UNDER REPORTING?}

${ }^{1} \mathrm{G}$ Oligbu, ${ }^{2} \mathrm{~L}$ Pay, ${ }^{2} \mathrm{M}$ Fallaha, ${ }^{3} \mathrm{~L}$ Ahmed, ${ }^{4} \mathrm{OE}$ Oligbu, ${ }^{5} \mathrm{C}$ Durojaiye. ${ }^{1}$ Paediatric Infectious Diseases Research Group, Institute for Infection and Immunity, St George's, University of London, UK; ${ }^{2}$ Department of Paediatrics, Imperial College London, UK; ${ }^{3}$ Department of Paediatrics, Northwick Park Hospital, London, UK; ${ }^{4}$ Accident and Emergency Department, Queen Elizabeth Hospital, Woolwich, London, UK; ${ }^{5}$ Department of Infection and Tropical Medicine, Royal Hallamshire Hospital, Sheffield, UK

\subsection{6/archdischild-2018-rcpch.290}

Introduction Since 2015, an outbreak of Zika Virus infection $(\mathrm{ZiKV})$ has been reported in over 30 countries. Emerging evidence suggest $\mathrm{ZikV}$ causes a spectrum of neurologic diseases both directly and by secondary autoimmunity. In pregnancy, $\mathrm{ZiKV}$ is a well-known cause of congenital brain abnormalities, including microcephaly. ${ }^{1}$ It has also been linked with GuillainBarre syndrome. ${ }^{2}$ However, its association with meningoencephalitis is not well described, especially in children.

Methods We undertook a systematic review of the English literature published from 1947 to August 2017 to evaluate the risk factors, distribution, pathogenesis, clinical presentation, management and outcomes of encephalitis following Zika virus infection. Data sources included MEDLINE, EMBASE, Cochrane library, and references within identified articles. We also searched the papers using the ISI web of knowledge, to identify relevant articles and conference proceedings.

Results We identified 167 potential studies, of which 16 were duplicates and 140 were excluded on the basis of title and abstracts (figure 1). There were 9784 with suspected ZiKV in all age groups. 1411 cases were confirmed ZiKV either by RT-PCR in plasma, CSF and urine, ELIZA or MRI. There were $26(1.8 \%)$ cases of meningoencephalitis. Among eight studies reporting clinical characteristics and outcomes of 16 cases, the mean age was 43 years (Range 19-81) and $75 \%$ $(n=12)$ of cases were male. 63\% $(n=10)$ of the cases required admission to ITU, with a crude case fatality rate of $12 \%$ $(n=2)$.

Conclusions and clinical implications Complications from ZiKV, although uncommon, may be severe. With international spread, clinicians need to be aware that $\mathrm{ZiKV}$ may be associated with meningoencephalitis. Although our review found a paucity of evidence of Zika virus associated meningoencephalitis in children, standardising the collection and reporting for individual cases across regions and countries would further allow meaningful analysis of the data collected, enabling monitoring of trends over time.

\section{British Paediatric Neurology Association}

\section{G299 NUSINERSEN (SPINRAZA) IS THE FIRST DRUG APPROVED FOR SPINAL MUSCULAR ATROPHY (SMA): INITIAL EXPERIENCE IN PATIENTS WITH SMA TYPE 1 TREATED IN THE EXPANDED ACCESS PROGRAM (EAP)}

${ }^{1,2} \mathrm{M}$ Scoto, ${ }^{2} \mathrm{M}$ Main, ${ }^{2} \mathrm{P}$ Munot, ${ }^{3} \mathrm{~L}$ Edel, ${ }^{3} \mathrm{~F}$ Abel, ${ }^{3} \mathrm{E}$ Chan, ${ }^{2} \mathrm{~A}$ Manzur, ${ }^{1,2} \mathrm{~F}$ Muntoni.
${ }^{1}$ Dubowitz Neuromuscular Centre, Great Ormond Street, UCL Institute of Child, London, UK;
${ }^{2}$ Dubowitz Neuromuscular Unit, Great Ormond Street Hospital for Children, London, UK;
${ }^{3}$ Respiratory Unit, Great Ormond Street Hospital for Children, London, UK

10.1136/archdischild-2018-rcpch.291

Aim to report the experience in patients with SMA type 1 treated in the Nusinersen expanded access program (EAP) in a single centre that have the largest UK cohort of patients with SMA type 1 (24 patients). Infantile SMA type 1 is the most common form of classic proximal 5q-SMA and also the most severe with inability to achieve independent sitting and limited life expectancy. SMA is caused by mutations in the survival motor neuron 1 (SMN1) gene resulting in insufficient SMN protein levels. Nusinersen (commercial name SPINRAZA) is a modified antisense oligonucleotide that, administered via consecutive intrathecal injections, modulates the splicing of the SMN2 mRNA transcript to include exon 7, thereby increasing the production of full-length SMN protein. Following the promising results of a large international phase II clinical trial (ENDEAR) nusinersen has received FDA and EMA approval and is currently offered under EAP in several European countries.

Methods The motor abilities of all patients recruited in the EAP were systematically assessed using a validated score of motor function developed specifically for patients with SMA type 1 (CHOP-intend scale) and the Hammersmith Infant Neurological Examination-part 2 (HINE-2 motor milestone assessment). Information on SMN2 copies was collected from the patients" medical records while data on nutrition and respiratory function was collected at each visit prior drug administration.

Results Twenty-one/24 patients with SMA type 1 (age range 3 months- 9 years) were recruited into the EAP from March 2017. One patient is under evaluation while two patients are already recruited into an open label trial using the same drug. To date, patients in the EAP tolerated well the lumbar puncture procedure performed using local anaesthetic and no side effects or adverse events related to the drug were noted. In $75 \%$ of the patients an improvement of the CHOP-Intend total score was observed after the 4th or 5th injection.

Conclusion Our data confirm the safety of intrathecal Spinraza treatment in SMA type 1 as well as a degree of motor function improvement. More longitudinal data is necessary to evaluate the long-term benefits, the respiratory muscle response and the best responders. 